Internist $2017 \cdot 58: 626$

DOI 10.1007/s00108-017-0228-x

Online publiziert: 7. April 2017

๑) Springer Medizin Verlag GmbH 2017

CrossMark
L. Reinhardt ${ }^{1,2} \cdot$ H. Eiffert ${ }^{3} \cdot$ G. Wulf ${ }^{4} \cdot$ P. Ströbel ${ }^{2,5} \cdot$ S. C. B. Bremer ${ }^{1,2} \cdot$ A. Amanzada' V. Ellenrieder ${ }^{1,2} \cdot$ A. Neesse ${ }^{1}$

${ }^{1}$ Klinik für Gastroenterologie und gastrointestinale Onkologie, Universitätsmedizin Göttingen, Göttingen, Deutschland

${ }^{2}$ Leberzentrum Göttingen, Universitätsmedizin Göttingen, Göttingen, Deutschland

${ }^{3}$ Institut für Medizinische Mikrobiologie, Universitätsmedizin Göttingen, Göttingen, Deutschland

${ }^{4}$ Klinik für Hämatologie und Medizinische Onkologie, Universitätsmedizin Göttingen, Göttingen, Deutschland

${ }^{5}$ Institut für Pathologie, Universitätsmedizin Göttingen, Göttingen, Deutschland

\section{Erratum zu: Spontane Remission einer HCV-Infektion nach autologer Stammzelltransplantation bei einem 58-jährigen Patienten}

\section{Erratum zu:}

Der Internist (2017)

DOI 10.1007/s00108-017-0206-3

In der vorläufigen Online-Version des Beitrags ist ein Fehler in der Aufzählung und Zuordnung der Institutionszugehörigkeiten aufgetreten. Die Redaktion bittet die oben stehenden Institutionszugehörigkeiten der Autoren zu beachten.

\section{Korrespondenzadresse}

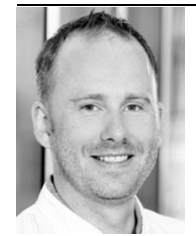

Dr. L. Reinhardt

Leberzentrum Göttingen, Universitätsmedizin Göttingen Robert-Koch-Str. 40, 37075 Göttingen, Deutschland lars.reinhardt@med.unigoettingen.de 